P073 ABSTRACT WITHDRAWN

\section{P074 PATIENTS WHO DEVELOP HEPATOCELLULAR CARCINOMA HAVE HIGHER RISK AMAP SCORES UP TO 5 YEARS BEFORE DIAGNOSIS}

Benjamin Giles*, Tushar Das, Zeshan Riaz, Joanna Dowman, Andrew Fowell, Richard Aspinall. Portsmouth Hospitals University NHS Trust, Portsmouth, UK

\subsection{6/gutjnl-2021-BASL.82}

Background Many patients with hepatocellular carcinoma (HCC) are diagnosed too late for potentially curative therapies and there is an unmet need for earlier detection. A recent international study assessed the 'aMAP' score (age, male gender, albumin-bilirubin, platelets) for stratifying chronic liver disease patients into low $(<0.2 \%)$, medium $(0.4-1 \%)$ or high (1.6-4\%) annual risk of developing HCC [Fan R et al, J Hepatol 2020]. However, the data was predominantly from patients with chronic viral hepatitis in tertiary centres, with potential for referral bias. The aim of our study was to assess whether aMAP scoring could have identified patients who developed HCC in an unselected UK population including non-viral liver diseases.

Methods A liver cancer database was used to identify all patients diagnosed with HCC over a 10 -year period at a major acute hospital serving a population of 675,000 . Clinical data included demographics, laboratory parameters, aetiology and stage of liver disease. The aMAP scores were calculated using blood tests from 1-5 years prior to HCC diagnosis to classify patients as low, medium or high risk.

Results We identified 226 patients diagnosed with HCC between 2010 and 2020. Median age at diagnosis was 72 years, $181(80 \%)$ were male and 215 (95\%) were of white ethnicity. Aetiological factors were alcohol 78 (35\%), non-alcoholic fatty liver disease 68 (30\%), chronic viral hepatitis 54 $(24 \%)$ and others 20 (9\%). There was no prior history of liver disease in $83(37 \%)$. Only 56 patients (25\%) were in a surveillance programme and just 47 (21\%) were Barcelona Clinic Liver Cancer (BCLC) stage A or lower at diagnosis.
However, a medium or high risk aMAP risk score was identifiable prior to HCC diagnosis in $99 \%$ at 1 year, $97 \%$ at 3 years and $96 \%$ at 5 years. A high risk aMAP score alone was found in $76 \%$ at 1 year, $73 \%$ at 3 years and in $67 \%$ at 5 years prior to diagnosis with HCC.

Conclusions Our study examined a 'real world' population, representative of the broad clinical spectrum of UK liver disease. We confirmed most HCC cases were diagnosed at a non-curative stage and were outside of formal surveillance. Importantly, we demonstrated the vast majority of patients with HCC have higher risk aMAP scores identifiable up to 5 years before diagnosis. Use of aMAP could improve targeting of HCC screening and our findings would support further, prospective evaluation in people with chronic liver disease in the UK.

\section{P075 UTILISING FRAX MODEL TO MANAGE BONE DISEASE (OSTEOPOROSIS) IN A NURSE-LED CLINIC FOR CIRRHOTIC PATIENTS}

Mohamed Elnagar*, Muhammad Saleem, Alison Beard, Samantha Whyld, Andrew Austin. Royal Derby Hospital, UK

\subsection{6/gutjnl-2021-BASL.83}

Introduction Bone disease (BD) is an important complication of chronic liver disease with prevalence between $12 \%-55 \%$ in cirrhotic patients. Osteoporosis is the most encountered BD type that may lead to fracture and is the only cirrhosis complication that remains after transplantation. Screening for BD using DEXA scan should be offered to all patient with established cirrhosis. Although FRAX model has not been studied on cirrhotic population, it may be a useful tool in the clinic setting to assess fracture risk with potential to reduce the use of DEXA scan.

Method A retrospective review was conducted of cirrhotic patients attending our stable cirrhosis clinic during the 1 st year of its establishment. Review included demographic data, aetiology of liver disease, height, weight and fracture incidents. FRAX score was calculated and used to triage the

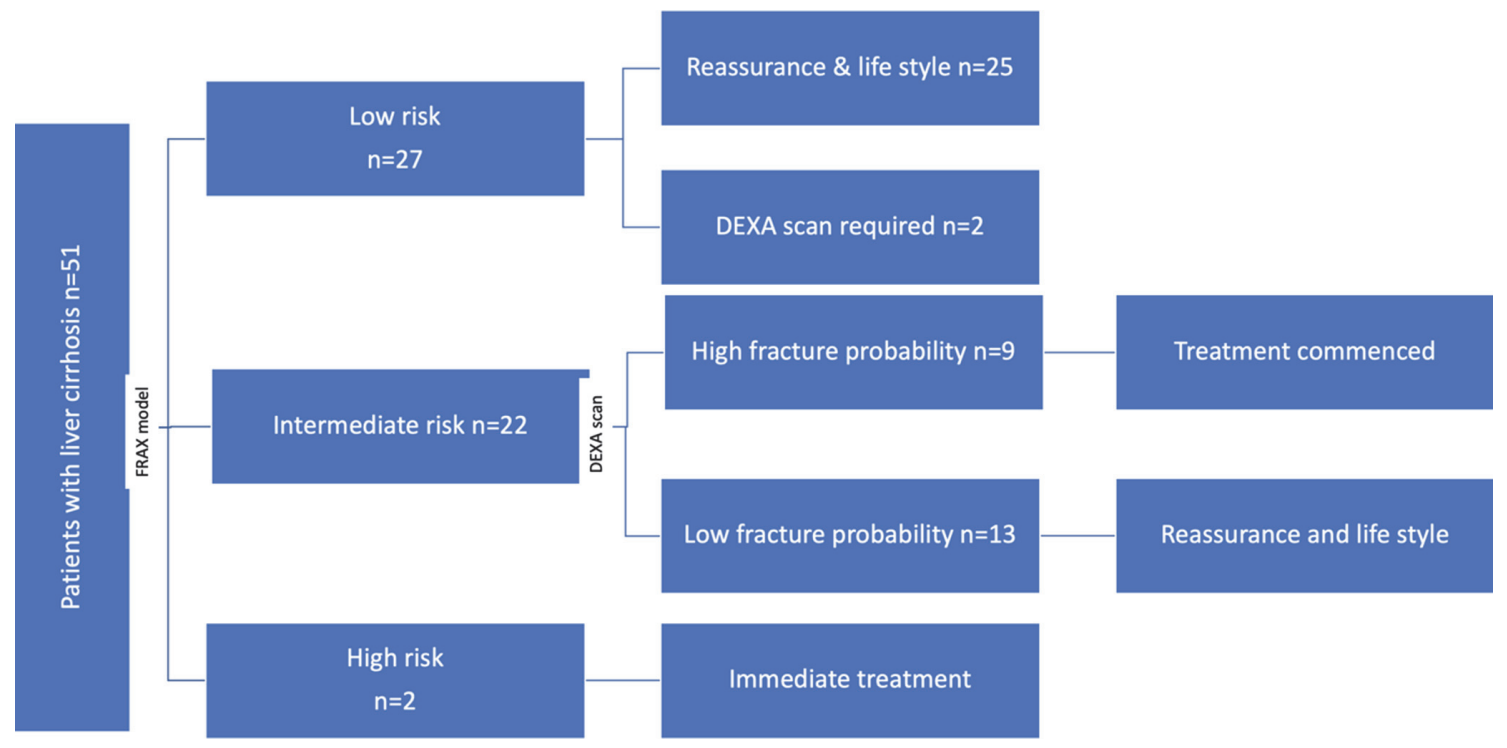

Abstract P075 Figure 1 Patients groups classified by FRAX model 
patients. For those who needed DEXA scan, FRAX score was re-calculated using the $\mathrm{T}$ score for neck of femur in order to identify those needing treatment.

Results In 2016, a total of 51(40 male) cirrhotic patients were reviewed in the clinic (ALD 25, NASH 9, other 17). Other aetiologies included HCV, PBC and HFE-related cirrhosis. Only 3 patients had Child Pugh B7 with the rest having Child A disease. FRAX score identified (27) low risk, (22) intermediate risk and (2) high risk for major fracture. Most of the low risk group were treated with reassurance and life style advice although 2 patients required DEXA scan due to radiological evidence of osteopenia and one of them was commenced on treatment. BMD was measured in patients with intermediate risk and 9 required intervention as their post DEXA scan FRAX score showed higher fracture probability. High risk patients were considered for treatment immediately without waiting for DEXA scan (figure 1). In all the three groups, treatment was achieved by consulting our rheumatology team. Fractures during our clinic period were reported in one of the high risk cohort following a fall while another patient has accidental spine fracture findings in a routine CXR. There were no fractures in the low or intermediate group over the 5 year follow up period.

Conclusions Using the FRAX model in patients with cirrhosis shows promise in the identification of low risk patients who do not require DEXA scan.

\section{P076 A MULTI-CENTRE, RANDOMIZED CONTROLLED STUDY, TO EVALUATE THE SAFETY AND PERFORMANCE OF THE DIALIVE LIVER DIALYSIS DEVICE IN PATIENTS WITH ACUTE ON CHRONIC LIVER FAILURE (ACLF) VERSUS STANDARD OF CARE (SOC) (ALIVER CONSORTIUM)}

\begin{abstract}
${ }^{1}$ Banwari Agarwal*, ${ }^{2}$ Faouzi Saliba, ${ }^{3,4}$ Dana Rodica Tomescu, ${ }^{5}$ Rafael Banares Canizares, ${ }^{6}$ Daniel Martin, ${ }^{7}$ Vanessa Stadlbauer, ${ }^{8,1}$ Gavin Wright, ${ }^{9}$ Mohammed Sheikh, ${ }^{10}$ Carrie Morgan, ${ }^{9}$ Fausto Andreola, ${ }^{9}$ Lennart Oettl, ${ }^{11}$ Carlos Alzola, ${ }^{8}$ Rahul Kumar, ${ }^{2}$ SophieCaroline Sacleux, ${ }^{6}$ Gernot Schilcher, ${ }^{12}$ Sebastian Koball, ${ }^{4}$ Andrada Tudor, ${ }^{13}$ Jaak Minten, ${ }^{14} \mathrm{Gema}$ Domenech, ${ }^{14}$ Juan Jose Aragones, ${ }^{9} \mathrm{Karl}$ Oettl, ${ }^{15}$ Katja Waterstradt, ${ }^{16}$ Stefanie M Bode-Boger, ${ }^{17}$ Luis Ibanez, ${ }^{1}$ Amir Gander, ${ }^{12,18}$ Jan Stange, ${ }^{19}$ Moises Sanchez, ${ }^{20}$ Rajeshwar P Mookerjee, ${ }^{9}$ Andrew Davenport, ${ }^{20}$ Nathan Davies, ${ }^{10}$ Marco Pavesi, ${ }^{21}$ Javier Fernandez, ${ }^{12,18}$ Steffen Mitzner, ${ }^{10}$ Vicente Arroyo, ${ }^{8}$ Rajiv Jalan. ${ }^{1}$ Royal Free Hospital, UK: ${ }^{2}$ AP-HP Hôpital Paul Brousse, Centre Hépato-Biliaire, INSERM unit No 1193, Université Paris-Saclay, France, ${ }^{3}$ Carol Davila University of Medicine and Pharmacy, Romania; ${ }^{4}$ Fundeni Clinical Institute Bucharest, Romania; ${ }^{5}$ Hospital Gregorio Maragnon, Spain; ${ }^{6}$ Peninsula Medical School, University of Plymouth, UK; 'Division of Gastroenterology und Hepatology Medical University of Graz, Austria; ${ }^{8}$ Basildon and Thurrock University Hospital, Mid and South Essex NHS Foundation Trust, Basildon, UK; ${ }^{9}$ University College London, UK; ${ }^{10}$ Yaqrit Ltd, UK; ${ }^{11}$ Etera Solutions, US; ${ }^{12}$ University Hospital Rostock, Germany; ${ }^{13}$ Independent Clinical Research Organisation, Belgium; ${ }^{14}$ Medical Statistics Core Facility IDIBAPS Hospital Clinic, Barcelona; ${ }^{15}$ Medlnnovation GmbH, Germany; ${ }^{16}$ Institut für Klinische Pharmakologie Magdeburg, Germany; ${ }^{17}$ Department of Gastroenterology, Gregorio Marañón General University Hospital, Health Research Institute Gregorio Marañón, Complutense University of Madrid, Spain; ${ }^{18}$ Fraunhofer IZI, Germany; ${ }^{19} I B M$, Ireland; ${ }^{20} / L D H$ - University College London, UK; ${ }^{21}$ Liver ICU, Liver Unit, Hospital Clinic Barcelona, Spain; ${ }^{22}$ Changi General Hospital, Singapore; ${ }^{23}$ EF-CLIF, Barcelona
\end{abstract}

10.1136/gutjnl-2021-BASL.84
Background and Aims Treatment of ACLF is an unmet need. DIALIVE is a novel liver dialysis device that replaces dysfunctional albumin and removes PAMPs and DAMPs. This RCT tests the hypothesis that DIALIVE improves the prognosis of ACLF patients. Primary endpoint was safety, and other endpoints included clinical and pathophysiologic effects and device performance.

Method EUH2020 funded the RCT. Patients population had ACLF Grades 1-3a, and the main endpoints were evaluated at Day 10. For the patient to be evaluable, they had 3-5 DIALIVE sessions. No specific hypothesis was to be statistically assessed. 2-populations were defined: Safety: Patients having at least 1 DIALIVE treatment. Modifiedsafety (MS): Evaluable patients. A post-hoc inferential Mixed Models for Repeated Measurements analysis was performed to evaluate the differences between groups at Days 5 and 10. Log-rank and Wald tests were performed to assess ACLF resolution.

Results Study: 32-ACLF patients with alcoholic cirrhosis were randomised either to DIALIVE $(\mathrm{N}=17 ; 13 \mathrm{M}$; age: 49 (12.4); CLIF-OFs: 10.3 (1.6); CLIF-C ACLFs: 48.6 (7.3)) or SOC $(n=15 ; 13 \mathrm{M}$; age: 49.1 (10.2); CLIF-OFs: 9.9 (1.2); CLIF-C ACLFs: 47 (6.5)). 30 patients (15 in each arm) comprised the MS set. DIALIVE was administered for a median of 3 sessions (range 1-5) in first 3-days (range 16) for a median of 8 hours (7-12) each day. Safety: 5 of 17 patients died in the DIALIVE group; 4 of 15 in SOC group. SAEs were seen in $64.7 \%$ in DIALIVE and $53.3 \%$ in the SOC group. Efficacy: CLIF-OF score: Significant improvement in the Liver $(p=0.045)$ and Brain $(p<0.001)$ subscores in DIALIVE; and deterioration of Lung subscore $(p=0.002)$ in the SOC group (Day 10). This resulted in a significant overall treatment effect in CLIF-OFs $(p=0.043)$. ACLF Grade: Significantly more cases reached ACLF 0 $(33.3 \%$ vs $66.7 \%)$ favoring DIALIVE (logrank $\mathrm{p}=0.0357)$ and a $2.8 \mathrm{x}$ faster time to ACLF 0 (Wald test $\mathrm{p}=0.059$ ) (figure 1). CLIF-C ACLF score: Difference of means (standard error) between DIALIVE and SOC at Day 10 was -5.4 (2.9) in favour of the DIALIVE group $(p=0.064)$. MELD score: Significantly lower in DIALIVE at both Days $5(\mathrm{p}=0.049)$ and $10(\mathrm{p}=0.028)$ vs SOC.

Conclusions DIALIVE is safe and significantly increases proportion of patients resolving ACLF and reduces time to resolution. The data justify late phase clinical trials.

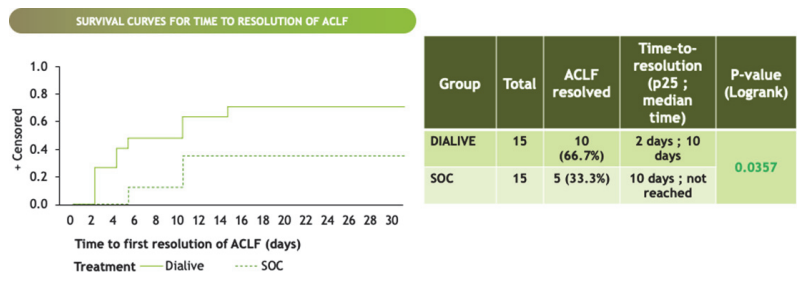

Abstract P076 Figure 1 This Kaplan Meier graph shows that significantly greater proportion of patients resolve ACLF with a faster resolution in the DIALIVE group 